\title{
Successful Treatment of Generalized Pustular Psoriasis of Pregnancy with Oral Cyclosporine
}

\author{
Zahra Najmi, MD, ${ }^{1}$ Ali Sayyadinejad, MD,${ }^{2}$ Narges Mohammadi, MD, ${ }^{3}$ Shahla Atrak, MD, ${ }^{1}$ Nader \\ Ghaffari, MD, ${ }^{4}$ Hamid Khederlou, MD ${ }^{5}$ \\ ${ }^{1}$ Department of Obstetrics and Gynecology, Zanjan University of Medical Sciences, Zanjan, Iran \\ ${ }^{2}$ Department of Dermatology, Zanjan University of Medical Sciences, Zanjan, Iran \\ ${ }^{3}$ Student Research Center, Zanjan University of Medical Sciences, Zanjan, Iran \\ ${ }^{4} \mathrm{MD}$, Anatomical and Clinical Pathologist, Booali Laboratory, Zanjan, Iran \\ ${ }^{5}$ Resident of Cardiology, Tehran Heart Center, Tehran University of Medical Sciences, Tehran, Iran
}

\section{ABSTRACT}

Generalized pustular psoriasis of pregnancy (GPPP) is a rare dermatosis that usually occurs during the third trimester of pregnancy. The diagnosis is primarily clinical, confirmed by laboratory investigations and histopathological typical findings. Here we report, a 26-year-old, gravida one woman, in 37 weeks of pregnancy, presenting with extensive erythematous, pruritic plaques with peripheral scaling and pustules. Patient was induced at $37^{+4}$ weeks and delivered a healthy $2900 \mathrm{~g}$ male infant. GPPP was eventually controlled with oral cyclosporine.

\section{INTRODUCTION}

Generalized pustular psoriasis of pregnancy (GPPP) or impetigo herpetiformis is a rare and severe dermatosis which usually occurs during the third trimester of pregnancy. ${ }^{1,2}$ It presents with generalized erythematous plaques, peripheral scales and non-infectious pustules symmetrically and grouped and often starting in the flexures and intertriginous areas and then spreads to the entire body. $2,3,4$ Many studies have suggested that pathogenesis is unknown. ${ }^{5}$ It can be associated with constitutional symptoms including fever, diarrhea, pain, gastrointestinal upset, malaise and arthralgia. ${ }^{2,6,7}$ Various factors play a role in triggering GPPP, such as topical therapy (coal tar, anthralin), pregnancy, infection, hypocalcemia, hypoparathyroidism and drugs. ${ }^{8}$ GPPP usually disappears after delivery, ${ }^{9}$ however, if untreated during pregnancy there is an increase in the risk of stillbirth. ${ }^{10,11}$ Complications include fluid and electrolyte imbalance such as hypocalcaemia and secondary infection, sepsis, intrauterine growth retardation, premature delivery and fetal abnormalities due to placental insufficiency. $6,12,13$ It is difficult to control the disease during pregnancy, especially when mother is unstable and there is no response to systemic steroids. ${ }^{9}$ We report a case of generalized pustular psoriasis of pregnancy, which was successfully treated with cyclosporine.

\section{CASE PRESENTATION}

A 26-year-old female, gravida 1, para 0, in 37 weeks of pregnancy, was referred to our tertiary center for a recently appearing and

May 2020 Volume 4 Issue 3 
progressive generalized skin eruption. She had extensive erythematous, pruritic plaques with peripheral scaling and pustules. On initial physical examination, the abdomen and back showed characteristic pustular eruption with erythema suggestive of pustular psoriasis of pregnancy (Figure 1a). The patient had no personal or family history of psoriasis and no relevant drug history. Antenatal examinations were carried out at regular intervals, with no found signs of placental insufficiency. Fetal movements and fetal heart rate were normal. Initially the patient was put on topical steroids (Triamcinolone) and told to use petrolatum. Oral antibiotics were administered to avoid secondary bacterial infection. Partial response was achieved in primary lesions after 3 days, but new pustular lesions appeared on the face, buttocks and limbs (Figure $1 \mathrm{~b}, \mathrm{c}, \mathrm{d}$ ). At $37^{+4}$ weeks, the patient had electrolyte imbalance and a fever. Because of the risk of stillbirth and perinatal mortality, labor was induced, and patient gave birth to a $2900 \mathrm{~g}$ male infant with a normal vaginal delivery. She was transferred to ICU. Cyclosporine was added in order to prevent the development of new lesions and elimination of prior lesions. Lab tests showed low levels of albumin, calcium and iron, thus Albumin 20\%, Ca-D and ferofolic were also begun.

\section{Culture}

Both the fungal and bacterial cultures of the pustular lesions were negative.

\section{Laboratory Investigations}

Blood analyses were carried out throughout the time of hospitalization. Laboratory tests showed leukocytosis of $19.200 / \mathrm{mm}^{3}$ with a raised erythrocyte sedimentation rate (ESR) and $C$ reactive protein (CRP). Serum levels of calcium, albumin and iron were low. Parathormone hormone, serum levels of sodium and potassium, rheumatologic tests, blood cultures and other laboratory investigations were within normal limits (or negative).

Figure 1. Extensive erythematous, pruritic plaques with peripheral scaling and sterile pustules on the abdomen and back, suggestive of pustular psoriasis of pregnancy (a). Pustular lesions on the face (b), buttocks (c) and limbs (d).

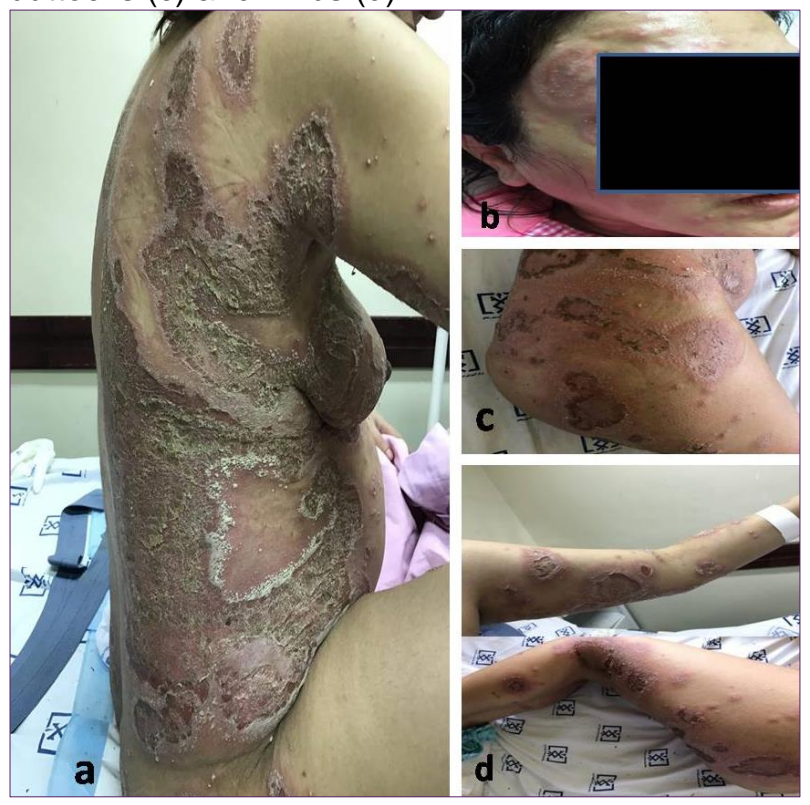

\section{Histopathology}

The histopathological findings included focal parakeratosis, acanthosis, elongated rete ridges, and subcorneal and intraepidermal spongiform pustules of Kogoj, containing neutrophils, munro microabscesses, as well as perivascular and papillary dermal infiltration of lymphocytes and neutrophils. Pathological diagnosis was pustular psoriasis of pregnancy (Figure 2).

\section{Outcome and Follow-Up}

Improvement of pustular psoriasis lesions began after 8 days treatment with cyclosporine. The patient presented with only residual hyperpigmentation and desquamation 17 days later (Figure 3). After resolution of cutaneous symptoms and hemodynamic stability, she was discharged from the hospital. 
Figure 2. (a) Subcorneal and intraepidermal spongiform pustules of Kogoj (HE; ×200).

(b) Papillary dermal infiltration of lymphocytes and neutrophils $(\mathrm{HE} ; \times 400)$.

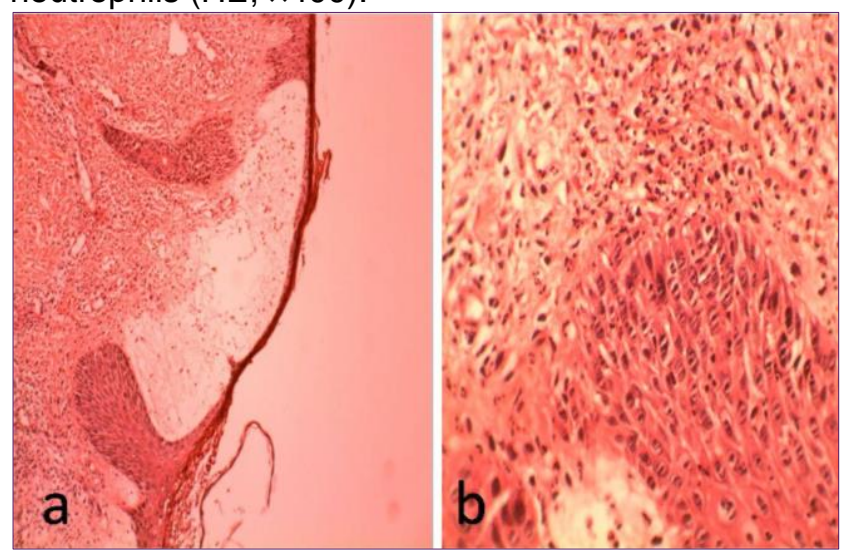

Figure 3. Healed lesions of pustular psoriasis with only residual hyperpigmentation and desquamation.

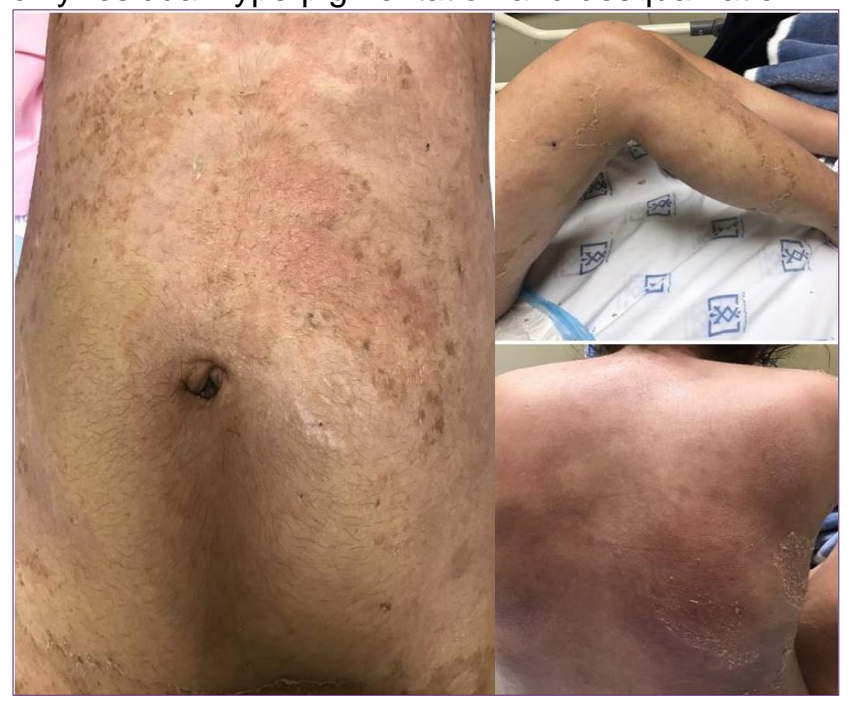

\section{DISCUSSION}

We report a case of generalized pustular psoriasis of pregnancy (GPPP) or impetigo herpetiformis with low levels of calcium, albumin and iron, raised ESR and CRP and leukocytosis, treated with topical corticosteroid and oral cyclosporine.

Psoriasis is a chronic disease with a mean prevalence of $2 \%$ worldwide. Clinical types have been classified: guttate, nail, inverted, vulgaris, keratodermic, pustular and erythrodermic. Pustular psoriasis can be localized or widespread, similar to our case in this study. ${ }^{10,11}$

GPPP is characterized by macroscopic pustules and is the most difficult variant of generalized pustular psoriasis to treat. 200 cases of GPPP have been reported up to now and it is still a matter of discussion regarding causes and treatment. ${ }^{2,3,9}$

Etiology is still unknown. Although it may be related to hypocalcemia, hypoparathyroidism, reduced epidermal antileukoproteinase activity and high progesterone in the third trimester of pregnancy. However, these findings can only be seen in a small number of patients. ${ }^{9,10,14}$

Prior studies have reported personal and family history of psoriasis in patients with GPPP. ${ }^{10,11}$

The diagnosis of GPPP primarily clinical with support from laboratory investigations and histopathological typical findings. Clinical examination is characterized by generalized erythematous plaques with peripheral scales and sterile pustules symmetrically and grouped and often starting in the flexures and intertriginous areas. Other signs and symptoms include poor general condition, fever, diarrhea, dehydration, tachycardia and seizures. Laboratory findings that may be detected are leukocytosis, raised ESR and $\mathrm{CRP}$, and negative bacterial and fungal cultures of pustules. Low calcium, phosphate and albumin and low parathyroid hormone levels are also common laboratory findings. Histopathological findings that confirm the diagnosis include parakeratosis, epidermal acanthosis and papillomatosis, elongated reteridges, neutrophilic inflammatory collections, intraepidermal microabscesses (spongiform pustules of Kogoj) and papillary 
dermal infiltration of lymphocytes. $4,6,9,10,11$ Early treatment of GPPP improves the prognosis for both the mother and fetus. The choice of treatment during pregnancy is systemic corticosteroids, with prednisone 30$60 \mathrm{mg} /$ day. Postpartum treatment is with cyclosporine. 2,6,15,16 Topical steroids are used to reduce cutaneous manifestations. ${ }^{2,17,18}$ Even if the pustules are non-infectious, antibiotics have been suggested to avoid secondary bacterial infection. ${ }^{6,10,11}$ Supportive treatment includes fluid and electrolyte balance, pain management and intensive care and fetal monitoring in case of emergency early delivery. ${ }^{5,19}$ In our case, topical steroids were initially begun along with antibiotic treatment. Because of the severity of the disease and risk to the fetus, labor was induced, and cyclosporine was added.

\section{Conflict of Interest Disclosures: None}

Funding: None

Corresponding Author:

Dr Narges Mohammadi

Student Research Center

Zanjan University of Medical Sciences

Zanjan, Iran

E-mail: $\underline{\text { dr.nargesmohammadi@yahoo.com }}$

\section{References:}

1. Luan L, Han S, Zhang Z, Liu X. Personal treatment experience for severe generalized pustular psoriasis of pregnancy: two case reports. Dermatol Ther. 2014 May-Jun;27(3):1747.

2. Shah A, Makhecha M. Pustular Psoriasis of Pregnancy with Acrodermatitis Continua of Hallopeau. Indian J Dermatol. 2016;61(1):123.

3. Hazarika D. Generalized pustular psoriasis of pregnancy successfully treated with cyclosporine. Indian J Dermatol Venereol Leprol. 2009;75(6):638.

4. Oumeish OY, Farraj SE, Bataineh AS. Some aspects of impetigo herpetiformis. Arch Dermatol 1982;118:103-5.
5. Nasser N, Sasseville D. Dermatologic diseases of pregnancy. Dermatologie - Conferences Scientifiques. 2006;5:1-6.

6. Shaw CJ, Wu P, Sriemevan A. First trimester impetigo herpetiformis in multiparous female successfully treated with oral cyclosporine. BMJ Case Rep. 2011;12:11-20.

7. Champion RH, Burton JL, Burns DA. Rook's Textbook of Dermatology. Sixth edition Oxford: Blackwell Scientific Publications. 1998;16:39-40.

8. Keerthi S, Rangaraj M, Karthikeyan K. Telmisartan aggravates pustular psoriasis. $J$ Pharmacol Pharmacother. 2015;6(2):107-9.

9. Patsatsi A, Theodoridis TD, Vavilis D, Tzevelekis V, Kyriakou A, Kalabalikis D, et al. Cyclosporine in the management of impetigo herpetiformis: a case report and review of the literature. Case Rep Dermatol. 2013;5(1):99-104.

10. Kondo RN, Araújo FM, Pereira AM, Lopes VC, Martins LM. Pustular psoriasis of pregnancy (impetigo herpetiformis)-case report. An Bras Dermatol. 2013;88(6):186-9.

11. Azulay-Abulafia L, Brotas A, Braga A, Volta AC, Gripp AC. Psoríase pustulosa da gestação (impetigo herpetiforme): relato de dois casos e revisão na literatura. Rev Bras Ginecol Obstet. 2004;26:153-159.

12. Vun YY, Jones B, Al-Mudhaffer M, Egan C. Generalized pustular psoriasis of pregnancy treated with narrowband UVB and topical steroids. J Am Acad Dermatol. 2006;54(2):28-30.

13. Bin Yap FB. Impetigo herpetiformis: A rare dermatosis of pregnancy. South Med J. 2009;102:1186-7.

14. Roth MM. Pregnancy dermatoses: diagnosis, management, and controversies. Am J Clin Dermatol. 2001;12:25-41

15. Brightman L, Stefanato CM, Bhawan J, Phillips TJ. Third-trimester impetigo herpetiformis treated with cyclosporine. J Am Acad Dermatol. 2007;56(2): 62-4

16. Martins GA, Arruda L. Systemic treatment of psoriasis - Part I: methotrexate and acitretin. An Bras Dermatol. 2004;79:263-278.

17. Okuno H, Ogura K, Okuyama R, Itoi E. Two cases of acrodermatitis continua of Hallopeau associated with generalized arthritis. Acta Dermatovenerol Croat. 2013;21:265-7

18. Imai $\mathrm{N}$, Watanabe $\mathrm{R}$, Fujiwara $\mathrm{H}$, Ito $\mathrm{M}$, Nakamura A. Successful treatment of impetigo herpetiformis with oral cyclosporine during pregnancy. Arch Dermatol. 2002;138(1):128-9.

19. Arslanpence I, Dede FS, Gokcu M, Gelisen O. Impetigo herpetiformis unresponsive to therapy in a pregnant adolescent. J Pediatr Adolesc Gynecol. 2003;16(3):129-132.

May 2020 Volume 4 Issue 3 